\title{
Improving half-projected spin-contaminated wave functions by multi-configuration perturbation theory
}

\author{
Zsuzsanna É. Mihálka, ${ }^{1}$ Ágnes Szabados, ${ }^{2}$ and Péter R. Surján*2 \\ 1)Laboratory of Theoretical Chemistry, Institute of Chemistry, Faculty of Science, \\ and George Hevesy Doctoral School of Chemistry, ELTE Eötvös Loránd University, H-1518 Budapest 112, P.O.B. 32, \\ Hungary \\ ${ }^{2)}$ Laboratory of Theoretical Chemistry, Institute of Chemistry, Faculty of Science, ELTE Eötvös Loránd University, \\ H-1518 Budapest 112, P.O.B. 32, Hungary
}

(*Electronic mail: peter.surjan@ttk.elte.hu)

\begin{abstract}
Allowing triplet components of individual geminals, spin-contaminated strongly orthogonal geminal wave functions may emerge, which can be ameliorated by spin-projection techniques. Of the latter, halfprojection was previously shown to be useful, offering a compromise between the amount of remaining spin-contamination and the violation of size-consistency generated by projection. This paper investigates how a half-projected spin-contaminated geminal wave function can be improved by multi-configuration perturbation theory, to incorporate dynamical correlation effects.
\end{abstract}

\section{INTRODUCTION}

Multi-reference perturbation theory (MRPT), aiming at correcting wave functions that consist of more than one determinant, has long been studied in detail and several versions of it have appeared (see, e.g. Refs. $\left.{ }^{1-11}\right)$. They often rely on some specific structure of the multi-reference function (e.g., complete active space $(\mathrm{CAS})^{12,13}$, generalized active space $(\mathrm{GAS})^{14}$, generalized valence bond $(\mathrm{GVB})^{15,16}$, antisymmetrized product of strongly orthogonal geminals (APSG) ${ }^{17,18}$ ), although correction schemes to general incomplete model spaces have also been developed ${ }^{19-21}$.

One general perturbation approach, which can, in principle, perturb reference states of any structure, is represented by the so-called multi-configuration perturbation theory (MCPT) ${ }^{22-25}$. In this paper, we shall apply its simplest form ${ }^{22}$, which will be reviewed in Sect.III for completeness.

The reference wave function, underlying the present perturbation treatment, has a rather complicated structure. It is based on a strongly orthogonal geminal ${ }^{26,27}$ wave function, in which however, individual geminals are not singlet constrained. Such wave functions have been studied by Rassolov ${ }^{28-30}$, by Head-Gordon ${ }^{31,32}$, as well as in our laboratory ${ }^{33-35}$. It was shown that allowing for triplet components improves quality of the strongly orthogonal geminal wave functions significantly, but at the same time, it possibly generates considerable spincontamination. The latter can be prevented by explicit spin-coupling ${ }^{33}$ following a valence-bond type philosophy, spin-adaptation ${ }^{36}$ via evaluating the effect of $\hat{S}^{2}$, or by spin-projection techniques ${ }^{34}$, similar to ones applied to the Unrestricted Hartree-Fock (UHF) wave functions ${ }^{37-39}$. Full spin-projection followed by variation according to the latter scheme was shown to generate significant size inconsistency. Therefore, in a previous paper, we investigated the performance of the so-called half-projection (HP) technique advocated by Smeyers ${ }^{40}$. This early method has recently been revisited and successfully applied within a mean-field theory ${ }^{41}$ as well as to geminal-based wave functions ${ }^{35}$. As shown in Ref. ${ }^{35}$ that half-projection, while significantly reduces spincontamination, generates much smaller size-consistency error than fully-projected spin-pure wave functions do.

Geminal-based reference states describe well static correlation, but need corrections to account for sufficient dynamic correlation. We aim at incorporating the latter in this paper by means of MCPT.

\section{HALF-PROJECTED UNRESTRICTED GEMINALS}

An $M_{S}=0$ geminal is written as

$$
\psi_{\mu}^{+}=\sum_{i j}^{(\mu)} C_{i j} i_{\beta}^{+} j_{\alpha}^{+},
$$

with Greek indices labeling geminals, Latin indices $i, j, k, \ldots$ referring to to spatial orbitals. To keep geminals strongly orthogonal, we assign disjoint sets of spatial orbitals to each ${ }^{26,27}$, as expressed by symbol $(\mu)$ on the summation. Unlike singlet constrained geminals, where the coefficient matrix $C$ is kept symmetric, we impose no symmetry for it, which may generate a triplet component corresponding to the anti-symmetric part of $C$. Geminal expansion coefficients $C_{i j}$ have been variationally optimized in each case, whether or not applying halfprojection.

In the present paper, spatial orbitals are the natural orbitals (NOs) of the $M_{S}=0$ UHF wave function (UNOs), and are not optimized, cf. UNO-CAS ${ }^{42}$. 
To express the fact that the geminals are expanded within the local subset of spatial orbitals assigned to them, we call them "strictly localized", and the entire $\mathrm{N}$-electron wave function is written as

$$
|\mathrm{USLG}\rangle=\prod_{\mu}^{N / 2} \psi_{\mu}^{+}|\mathrm{vac}\rangle .
$$

In the above acronym, ' $U$ ' refers to the application of UNOs.

Eventual spin-contamination exhibited by Eq.(2) has to be treated. For reasons mentioned above, we employ half-projection ${ }^{40,43}$, achieved by applying the projection operator

$$
\hat{P}_{S}=\frac{1}{2}\left(1+f \hat{\mathscr{P}}_{\mathrm{sf}}\right),
$$

where $f=(-1)^{S} S$ being the desired overall spin quantum number, and $\hat{\mathscr{P}}_{\text {sf }}$ standing for the spin-flip operator acting as

$$
\begin{array}{r}
\hat{\mathscr{P}}_{\mathrm{sf}} i_{\beta}^{+} \ldots j_{\beta}^{+} k_{\alpha}^{+} \ldots l_{\alpha}^{+}|\mathrm{vac}\rangle \\
=k_{\beta}^{+} \ldots l_{\beta}^{+} i_{\alpha}^{+} \ldots j_{\alpha}^{+}|\mathrm{vac}\rangle \\
=(-1)^{N / 2} i_{\alpha}^{+} \ldots j_{\alpha}^{+} k_{\beta}^{+} \ldots l_{\beta}^{+}|\mathrm{vac}\rangle
\end{array}
$$

where $N / 2=N_{\alpha}=N_{\beta}$ (merely $S_{z}=0$ states are treated here).

Our final normalized reference state has the form

$$
|\mathrm{HP}-\mathrm{USLG}\rangle=\frac{\hat{P}_{S}|\mathrm{USLG}\rangle}{\left\langle\hat{P}_{S} \mathrm{USLG} \mid \hat{P}_{S} \mathrm{USLG}\right\rangle^{1 / 2}} .
$$

As known, half-projection by operator (3) does not remove all contaminants, e.g. for even $S$ values the components with odd $S$ are eliminated. For technical details, we refer to $\operatorname{Ref}^{35}$.

There are two philosophies by which spincontaminated wave functions can be subjected to a perturbation treatment. One is to follow the route used in inter-molecular interactions, when antisymmetry of the product function is violated at the zeroth order, and symmetry-adapted perturbation theory (SAPT) is used to restore symmetry ${ }^{44-47}$. In this method the actual reference state is the unprojected, symmetry-violating wave function of Eq.(2), and symmetry-projection is applied in course of the perturbative treatment. Such a solution has been generalized to the present, spinviolating situation, as will be published in a separate paper. The second possibility followed here, is to treat the projected wave function directly by some flexible variant of perturbation theory. As stated before, MCPT is ideally suited for this purpose.

The role of (half-)projection in extended systems has to be commented. It is known for a long time ${ }^{48,49}$ that the effect of full projection diminishes with increasing system size, for energy per monomer. In a recent paper ${ }^{35}$, we have also addressed this point and found that the same holds for half-projection, too. These findings stand for the energy of the system, the structure of the wave function, however, is affected by the projection.

\section{REVIEW OF MCPT}

Rather than being one specific method, MCPT is a general framework which falls into the "diagonalizethen-perturb" ("single-but-multi") category of multireference perturbation theories.

Define a projector corresponding to the normalized $\mid$ HP-USLG $\rangle$ wave function

$$
\hat{O}=\mid \text { HP-USLG }\rangle\langle\text { HP-USLG }|
$$

and its orthogonal complement $\hat{P}=1-\hat{O}$. Next, consider a set of determinants $|k\rangle$, generated by single, double, etc. excitations on a dominant determinant $|\mathrm{D}\rangle$ chosen as the 'principal' component of $|\mathrm{HP}-\mathrm{USLG}\rangle$ :

$$
|\mathrm{HP}-\mathrm{USLG}\rangle=d_{0}|\mathrm{D}\rangle+\sum_{k=1} d_{k}|k\rangle
$$

where only the singular case $d_{0} \sim 0$ is excluded. In some cases, e.g. when addressing open-shell triplet states, $|D\rangle$ can be substituted by the respective spin-adapted configuration-state-function (CSF).

Consider now the set of vectors $\mid$ HP-USLG $\rangle$ and $|k\rangle$ $(k=1,2, \ldots)$, which altogether span the full $N$-electron space. However, they constitute an overlapping basis, as $\mid$ HP-USLG $\rangle$ and vectors $|k\rangle$ are not orthogonal. For brevity, we use $|0\rangle \equiv \mid$ HP-USLG $\rangle$ in the following. To deal with the situation of overlapping basis vectors, consider the set of projected determinants

$$
\left|k^{\prime}\right\rangle=\hat{P}|k\rangle=(1-\hat{O})|k\rangle
$$

which are orthogonal to the reference state, but overlap among themselves. The full overlap matrix reads

$$
\mathscr{S}=\left[\begin{array}{cc}
\langle 0 \mid 0\rangle & \langle 0|\hat{P}| l\rangle \\
\langle k|\hat{P}| 0\rangle & \langle k|\hat{P}| l\rangle
\end{array}\right]=\left[\begin{array}{cc}
1 & \mathbf{0} \\
\mathbf{0} & \mathbf{S}
\end{array}\right]
$$

where the overlap matrix $\mathbf{S}$ of the projected excited determinants possesses the elements

$$
S_{k l}=\left\langle k^{\prime} \mid l^{\prime}\right\rangle=\langle k|\hat{P}| l\rangle=\delta_{k l}-\langle k \mid 0\rangle\langle 0 \mid l\rangle=\delta_{k l}-d_{k} d_{l}
$$

having the inverse

$$
\left(S^{-1}\right)_{k l}=\delta_{k l}+\frac{d_{k} d_{l}}{1-\sum_{j=1}\left(d_{j}\right)^{2}}=\delta_{k l}+\frac{d_{k} d_{l}}{d_{0}^{2}} .
$$


Knowing the inverse metric explicitly permits one to specify a reciprocal set of vectors $\left\langle\tilde{k}^{\prime}\right|$ :

$$
\left\langle\tilde{k}^{\prime}\right|=\sum_{j=1}\left(S^{-1}\right)_{k j}\left\langle j^{\prime}\right|,
$$

which, using Eq.(7), can be written as

$$
\left\langle\tilde{k}^{\prime}\right|=\left\langle k^{\prime}\right|+\frac{d_{k}}{d_{0}^{2}} \sum_{j=1} d_{j}\left\langle j^{\prime}\right|
$$

which are bi-orthogonal to the original basis vectors, and facilitate the following definition of a zero order Hamiltonian

$$
\hat{H}^{0}=E_{0} \hat{O}+\sum_{k=1} E_{k}\left|k^{\prime}\right\rangle\left\langle\tilde{k}^{\prime}\right|,
$$

where $E_{0}=\langle 0|H| 0\rangle$ is chosen as the energy of the reference state. The excited state energies $E_{k}$ are in principle arbitrary quantities that define the partitioning via specifying $\hat{H}^{0}$.

Important properties of $\hat{H}^{0}$ are:

$$
\hat{H}^{0}|0\rangle=E_{0}|0\rangle
$$

and

$$
\hat{H}^{0}\left|k^{\prime}\right\rangle=E_{k}\left|k^{\prime}\right\rangle \text {. }
$$

The lowest order energies, as obtained by biorthogonal perturbation theory, can be expressed as follows. With $\hat{W}=\hat{H}-\hat{H}^{0}$ standing for the perturbation operator, at first order we have

$$
E^{1}=\langle 0|\hat{W}| 0\rangle=0,
$$

while the second order energy reads

$$
E^{2}=-\langle 0|\hat{W} \hat{Q} \hat{W}| 0\rangle=-\sum_{k=1} \frac{\left\langle 0|\hat{W}| k^{\prime}\right\rangle\left\langle\tilde{k}^{\prime}|\hat{W}| 0\right\rangle}{E_{k}-E_{0}} .
$$

In this formula $E_{0}$ is the energy of the reference state, while the excited energies are chosen as the diagonal matrix elements $E_{k}=\langle k|\hat{H}| k\rangle$, in the spirit of the EpsteinNesbet (EN) partitioning ${ }^{50,51}$.

The simplicity of these results has the consequence that with MCPT one can perturb reference functions of arbitrary complexity and structure. All that is needed is a list of configurations, i.e., the weights of the of determinants in the reference function. It is, therefore, well suited for evaluating perturbations of the HP-USLG reference state. A few examples will be reported below.

Neither the zero-order projected wave function, nor the MCPT procedure applied here, are fully size extensive/consistent. While a variant of MCPT has been previously published which is size-consistent at the second $\operatorname{order}^{23}$, here we remained at the formulation discussed here, because the HP-USLG reference state would spoil size consistency anyway. To demonstrate the numerical effect of this error, we performed a test calculation, see Sect. IV IV.1.

\section{RESULTS}

As mentioned in Sect. II, in obtaining the numerical results presented below, the underlying basis set was set up by UNOs. Geminal subspaces were determined according to the pairing of UNOs, with two-dimensional active geminals built from two orbitals with occupation numbers adding up to two. After half-projection, we switched to the respective NOs, while doubly occupied and virtual orbitals were pseudo-canonicalized by diagonalizing the corresponding block of the generalized Fock matrix.

\section{IV.1. The $\mathrm{H}_{4}$ model}

We considered first the frequently investigated model of four $\mathrm{H}$ atoms, monitoring its rectangle-to-square distortion near the square arrangement. The four atoms move on a circle of 1.0 bohr radius. The actual geometry is specified by angle $\alpha=\angle(\mathrm{HXH})$, with $\mathrm{X}$ denoting the center of the circle. Each of the two geminals is correlated at the (HP-)USLG level of theory. In the geometry range presented here, the system has a triplet ground state based on full configuration interaction (FCI). USLG calculations yield spin contaminated results for the ground state, with $\left\langle\hat{S}^{2}\right\rangle_{\text {USLG }}$ falling between 1.43 and 1.80 (instead of being 2.00 for spin pure cases). The singlet state can be described in a spin pure manner by USLG. In comparison, HP-USLG yields a pure triplet state, but a slightly spin contaminated singlet

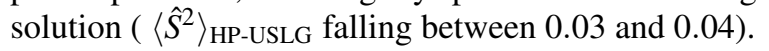

Figures 1-3. show the energies of the triplet state, singlet sate, and the singlet-triplet gap, respectively. For two selected angles, the triplet energy and the gap is given also in Table I., to show the numbers more accurately. For benchmark, the FCI data were used in each case.

The results in Table I indicate that unrestricted geminal energies are far from being sufficiently accurate, and half-projection offers only a marginal improvement. If MCPT is applied onto the USLG reference state, the results do not get better. However, if MCPT is used to correct the half-projected USLG wave function, both total energies and energy differences greatly improve, and the curves in Figs. 1-3. run nicely parallel with the FCI ones.

In Table II, we present the energies of a single $\mathrm{H}_{4}$ system and its dimer, with monomers separated at infinite distance in the latter, in order to test the size-consistency of the HP-USLG reference state and of the second order PT correction as obtained by MCPT. As seen, the 28 $\mathrm{mE}_{\mathrm{h}}$ size consistency error of the reference state is diminished by cca $15 \%$ upon computing the second order PT correction. Note that if the PT series converged, the 


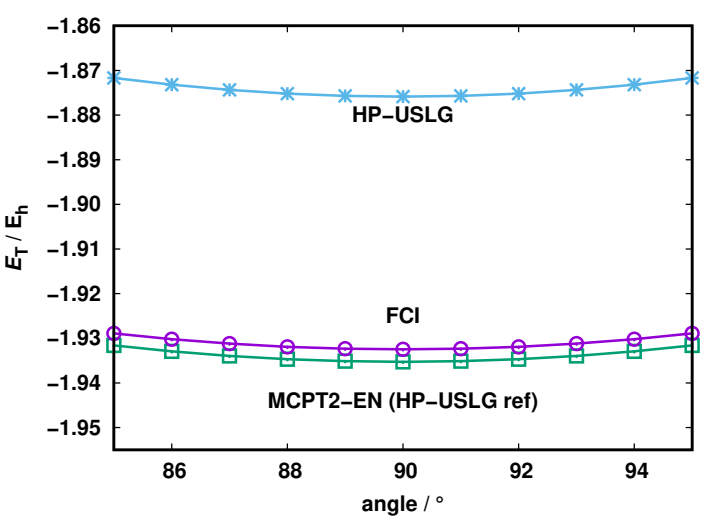

FIG. 1. Total energy (in $E_{h}$ ) of the triplet state of the $\mathrm{H}_{4}$ molecule in $6-311 \mathrm{G}^{* *}$ basis. Term "EN" refers to the EpsteinNesbet type partitioning of MCPT.

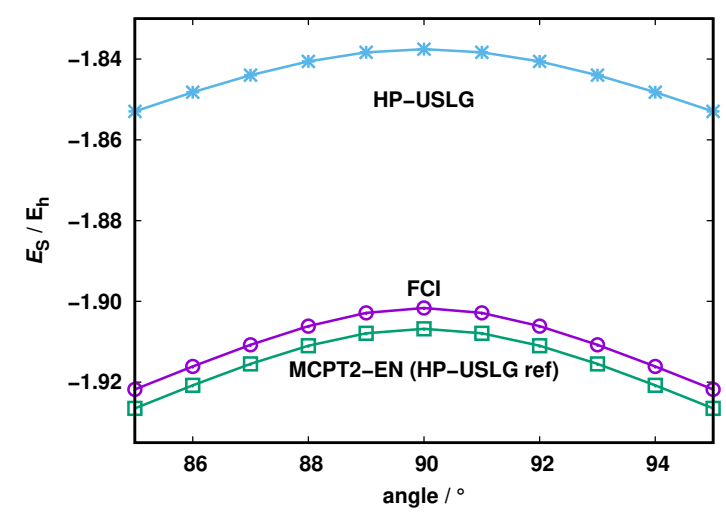

FIG. 2. Total energy (in $\mathrm{E}_{\mathrm{h}}$ ) of the singlet state of the $\mathrm{H}_{4}$ molecule in $6-311 \mathrm{G}^{* *}$ basis.

TABLE I. Total energy of the triplet state (in $\mathrm{E}_{\mathrm{h}}$ ) and singlettriplet gap $\left(\Delta E=E_{\mathrm{S}}-E_{\mathrm{T}}\right.$, in $\left.\mathrm{mE}_{\mathrm{h}}\right)$ of the $\mathrm{H}_{4}$ system in 6$311 \mathrm{G}^{* *}$ basis. Benchmark is provided by FCI.

\begin{tabular}{|l|cc|cc|}
\hline & \multicolumn{4}{|c|}{$\alpha /{ }^{\circ}$} \\
\hline method & $E_{\mathrm{T}}$ & $\Delta E$ & $E_{\mathrm{T}}$ & $\Delta E$ \\
\hline USLG & -1.863433 & 20.41 & -1.864476 & 39.53 \\
USLG-MCPT & -1.930691 & -3.77 & -1.983307 & 67.08 \\
\hline HP-USLG & -1.871671 & 18.72 & -1.875856 & 38.30 \\
HP-USLG-MCPT & -1.931599 & 5.11 & -1.935273 & 28.48 \\
\hline FCI & -1.928906 & 7.13 & -1.932478 & 30.84 \\
\hline
\end{tabular}

size-consistency error should naturally tend to zero with

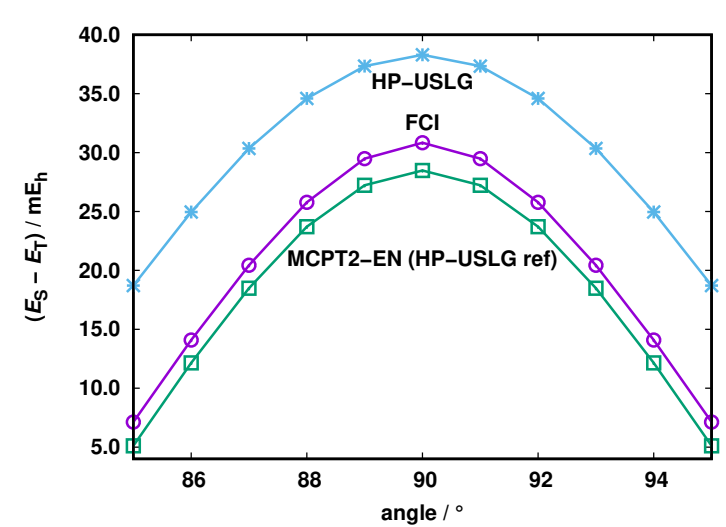

FIG. 3. Vertical excitation energies $\left(E_{\mathrm{S}}-E_{\mathrm{T}}\right.$, in $\left.\mathrm{mE}_{\mathrm{h}}\right)$ of the $\mathrm{H}_{4}$ molecule in $6-311 \mathrm{G}^{* *}$ basis.

increasing order. There are two alternative, energetically equivalent forms of the half-projected geminal product wavefunction of the dimer, differing in a spin flip of one of the monomers. The PT corrected function and energy can be seen to be the same when built upon either of these two possible zero order functions.

TABLE II. Size consistency test of HP-USLG-based second order MCPT method for the $\mathrm{h}_{4}$ system in the singlet state. Total energies in Hartree, energy differences in $\mathrm{mE}_{\mathrm{h}}$. The monomer geometry is slightly distorted from the square arrangement with $\alpha=85^{\circ}$. The basis set is $6-311 \mathrm{G}^{* *}$.

\begin{tabular}{|l|cc|c|}
\hline method & $E_{\mathrm{H}_{4}}$ & $E_{\mathrm{H}_{8}}$ & $E_{\mathrm{H}_{8}}-2 E_{\mathrm{H}_{4}}$ \\
\hline HP-USLG & -1.852955 & -3.733701 & -0.028 \\
HP-USLG-MCPT2 & -1.926492 & -3.876795 & -0.024 \\
\hline
\end{tabular}

\section{IV.2. Ozone}

As a next example the $\mathrm{O}_{3}$ molecule was treated in the cc-pCVDZ basis $^{52}$. Of the 12 geminals constituting the product wave function, 6 are correlated both for USLG and HP-USLG: two have $\pi$ character, and four have $\sigma$ symmetry. Occupation number threshold for doubly occupied orbitals is 1.9996. At the USLG level of theory, the singlet state is not spin pure, with $\left\langle\hat{S}^{2}\right\rangle_{\mathrm{USLG}}=0.014$. This slight spin contamination is accompanied by spatial symmetry violation. The triplet state by USLG is of correct spin and spatial symmetry. HP-USLG is somewhat spin contaminated for both states, yielding $\left\langle\hat{S}^{2}\right\rangle_{\mathrm{HP}-\mathrm{USLG}}=0.023$ for the singlet, and 2.001 for the triplet solution, however, both states possess correct spatial symmetry. Since spin-contamination at the level of USLG is marginal, it is not expected to 
undermine the performance of PT built upon it ${ }^{53}$. (This is in marked difference with the case of p-benzyne and cyclobutadiene, vide infra.)

The ground state energy and the singlet-triplet gap is given in Table III. The present results are compared to linear-response coupled cluster (CCSD-LR or CCSDTLR) literature data by Jagau and Gauss ${ }^{54}$. The table shows that the total energy of the singlet state, as obtained by USLG, is rather far from the reference energy, but MCPT nicely improves it. The same holds for the vertical excitation energies, too. Half-projection induces only a minor improvement on the spin-contaminated singlet energy, while does not improve the excitation energy at all. Second order MCPT after half-projection shows rather similar results to the perturbed unprojected numbers.

TABLE III. Ground state energy (in $\mathrm{E}_{\mathrm{h}}$ ) and vertical excitation energy (in $\mathrm{eV}$ ) of the lowest triplet state of ozone in ccpCVDZ basis. Single-reference CC based linear response calculations are cited as benchmark. Symmetry of the molecule is $C_{2 v}$ with parameters taken from Ref. $^{54}$ as $R_{\mathrm{O}-\mathrm{O}}=1.2569 \AA$ and $\angle(\mathrm{OOO})=116.54^{\circ}$.

\begin{tabular}{|lcc|}
\hline \multicolumn{1}{c}{${ }^{1} A_{1}$} & ${ }^{1} A_{1} \rightarrow{ }^{3} B_{2}$ \\
\hline USLG & -224.3653 & 0.977 \\
USLG-MCPT & -224.9275 & 1.721 \\
\hline HP-USLG & -224.3746 & 0.937 \\
HP-USLG-MCPT & -224.9186 & 1.742 \\
\hline CCSD-LR $^{1}$ & -224.9981 & 1.421 \\
CCSDT-LR $^{1}$ & -225.0311 & 1.716 \\
\hline \multicolumn{1}{l}{${ }^{1}$ Ref. $^{54}$}
\end{tabular}

\section{IV.3. Para-benzyne, $\mathrm{C}_{6} \mathrm{H}_{4}$}

We continue with para-benzyne (Table IV). For reference, spin-flip calculations by Slipchenko and Krylov ${ }^{55}$ are used, the vertical gap of which is in nice agreement with the experimental value. Apart from the twoelectron unit with diradical character (built from $\sigma$ orbitals), three geminals (consisting of the $\pi$ orbitals of the carbon atoms) are also correlated at the level of USLG and HP-USLG. Here the role of half-projection appears to be much more important than for $\mathrm{O}_{3}$, since the unprojected perturbed USLG-MCPT gap is of wrong sign. The good agreement between unprojected USLG gap and the reference value is fortuitous, especially since the singlet USLG energy is quite far from the reference value. MCPT gives rather large second order corrections, but without half-projection it does so in an unbalanced manner, reversing the order of the singlet and triplet states. This error is nicely corrected if half-projection is applied before perturbation. Note also that the energy separa-
TABLE IV. Total energies (in $E_{\mathrm{h}}$ ) and adiabatic gap $\left(\Delta E=E\left({ }^{3} B_{1 u}\right)-E\left({ }^{1} A_{g}\right)\right.$ in $\mathrm{eV}$ ) for para-benzyne in $6-31 \mathrm{G}^{*}$ basis.

\begin{tabular}{|l|ccc|}
\hline & $E\left({ }^{1} A_{g}\right)$ & $E\left({ }^{3} B_{1 u}\right)$ & $\Delta E$ \\
\hline USLG & -229.42265 & -229.41751 & 0.140 \\
USLG-MCPT & -230.17913 & -230.19382 & -0.400 \\
\hline HP-USLG & -229.43891 & -229.43906 & -0.004 \\
HP-USLG-MCPT & -230.17014 & -230.16269 & 0.203 \\
\hline SF-OD & & & 0.174 \\
expt. & -230.15415 & & $0.165 \pm 0.016$ \\
\hline$\left\langle\hat{S}^{2}\right\rangle_{\text {HP-USLG }}$ & & & \\
$\left\langle\hat{S}^{2}\right\rangle_{\text {USLG }}$ & 0.25 & 2.17 & \\
\hline
\end{tabular}

${ }^{1}$ Spin-flip orbital optimized CC doubles, Ref. ${ }^{55}$

tion for para-benzyne is smaller by an order of magnitude than that for ozone.

Inadequacy of unprojected results is indicated by the expectation values of the $\hat{S}^{2}$ operators, which is quite pathological for the spin-contaminated singlet state without projection (the triplet state is spin-pure in this case.) After half-projection the spin of the singlet state improves greatly, while a small spin-contamination is generated for the triplet.

\section{IV.4. Cyclobutadiene, $\mathrm{C}_{4} \mathrm{H}_{4}$}

In our final example we asses the adiabatic gap between the rectangular singlet ground state and the lowest lying triplet excited state at equilibrium square geometry of cyclobutadiene. Two $\pi$-type geminals are allowed to be correlated (occupation number threshold for doubly occupied UNOs being 1.98) at the level of the reference. Calculations were performed in the aug'cc-pVTZ basis (prime indicating that the $f$ functions on carbon and $d$ functions on hydrogen atoms were dropped from the basis set). Geometric parameters for the rectangular singlet state, borrowed from Ref. ${ }^{56}$ are $R_{\mathrm{C}-\mathrm{C}}=1.562 \AA, R_{\mathrm{C}=\mathrm{C}}=1.349 \AA, R_{\mathrm{C}-\mathrm{H}}=1.077 \AA$ and $\angle(H C C)=134.9^{\circ}$ being the angle between $\mathrm{C}-\mathrm{H}$ and the longer $\mathrm{C}-\mathrm{C}$ bond. For the triplet state of $D_{4 h}$ symmetry, $R_{\mathrm{C}-\mathrm{C}}=1.442 \AA$ and $R_{\mathrm{C}-\mathrm{H}}=1.076 \AA$ were employed. The singlet root by USLG is heavily spin contaminated, as is reflected by the corresponding total spin expectation value in Table V. Just like for para-benzyne, spin symmetry violation is accompanied by breaking of spatial symmetry. HP-USLG greatly improves spin character of the singlet state and fully restores spatial symmetry. The triplet state is spin pure by both methods. Note however, that spin is correct by HP-USLG only after projection; the underlying geminal product function is in fact spin contaminated, i.e., it differs from the USLG solution. This explains the different energies obtained for the triplet state by USLG and HP-USLG (cf. Table V). Re- 
TABLE V. Total energies (in $\mathrm{E}_{\mathrm{h}}$ ) and adiabatic gap $\left(\Delta E=E\left({ }^{3} A_{2 g}\right)-E\left({ }^{1} A_{g}\right)\right.$ in $\left.\mathrm{kcal} / \mathrm{mol}\right)$ for cyclobutadiene in aug'-cc-pVTZ basis.

\begin{tabular}{|l|ccc|}
\hline & $E\left({ }^{1} A_{g}\right)$ & $E\left({ }^{3} A_{2 g}\right)$ & $\Delta E$ \\
\hline USLG & -153.71182 & -153.69196 & 12.5 \\
USLG-MCPT & -154.35800 & -154.36759 & -6.02 \\
\hline HP-USLG & -153.72581 & -153.72816 & -1.48 \\
HP-USLG-MCPT & -154.34365 & -154.30809 & 22.3 \\
\hline MR-AQCC/SA-CAS & -154.3658 & -154.3440 & 13.7 \\
\hline$\left\langle\hat{S}^{2}\right\rangle_{\text {HP-USLG }}$ & 0.21 & 2.00 & \\
$\left\langle\hat{S}^{2}\right\rangle_{\text {USLG }}$ & 0.74 & 2.00 & \\
\hline
\end{tabular}

${ }^{1}$ Ref. ${ }^{56}$, without zero-point energy

sults obtained by multi-reference averaged quadratic CC (MR-AQCC) $)^{56}$ serve as benchmark.

Energetic data reflect very similar tendencies to the case of para-benzyne. While adiabatic gap by USLG is remarkably accurate, that is merely accidental, as total energies are far from the reference MR-AQCC values. Applying MCPT with USLG reference deteriorates these results and provides an incorrect energetic order of states. In comparison, without PT, HP-USLG does not perform very well: it yields an incorrect sign for the singlet-triplet gap. Incorporating dynamic correlation by MCPT cures this problem, and provides a gap of correct sign and order of magnitude.

\section{CONCLUSION}

Severe spin-contamination of the reference state spoils the performance of PT. This well-known experience at the single-reference level is demonstrated here on multideterminantal examples. Pathological cases are seen to be remedied by applying half-projection prior to PT treatment. The generally applicable MCPT framework is ideally suited for incorporating dynamical correlation with the HP-USLG reference, and performs remarkably well in the EN-type partitioning.

\section{Data availability}

The data that support the findings of this study are available from the corresponding author upon reasonable request.

\section{ACKNOWLEDGMENTS}

This work was completed in the ELTE Institutional Excellence Program (TKP20 20-IKA-05) financed by the Hungarian Ministry of Human Capacities.

Zs.É.M. acknowledges the financial support of the ÚNKP-20-4 grant (New National Excellence Program provided by the Hungarian Ministry for Innovation and
Technology from the source of the National Research, Development and Innovation Fund).

${ }^{1}$ P. M. Kozlowski and E. R. Davidson. Chem. Phys. Letters, 222:615620, 1994.

${ }^{2}$ F. Chen, E. Davidson, and S. Iwata. Int. J. Quantum Chem., 86:256, 2002.

${ }^{3}$ D. Pahari, S. Chattopadhyay, S. Das, and D. Mukherjee. Chem. Phys. Letters, 381:223, 2003.

${ }^{4}$ H. Nakano. J. Chem. Phys., 99:7983, 1993.

${ }^{5}$ R. J. Cave and E. R. Davidson. J. Chem. Phys., 88:5770, 1993

${ }^{6}$ R. K. Chaudhuri and K. F. Freed. J. Chem. Phys., 107:6699, 1997.

${ }^{7}$ K. Hirao. Chem. Phys. Letters, 201:59, 1993.

${ }^{8}$ C. Angeli, R. Cimiraglia, S. Evangelisti, T. Leininger, and J.-P. Malrieu. J. Chem. Phys., 114:10252-10264, 2001.

${ }^{9}$ Y. Khait, J. Song, and M. Hoffmann. J. Chem. Phys., 117:4133-4145, 2002.

${ }^{10}$ M. R. Hoffmann, D. Datta, S. Das, D. Mukherjee, Á.. Szabados, Z. Rolik, and P. R. Surján. J. Chem. Phys., 131:204104, 2009.

${ }^{11}$ P. Jeszenszki, P. R. Nagy, T. Zoboki, A. Szabados, and P. R. Surján. Int. J. Quantum Chem., 114:1048, 2014.

${ }^{12}$ K. Andersson, P.-Å. Malmqvist, B. O. Roos, A. J. Sadlej, and K. Wolinski. J. Phys. Chem., 94:5483, 1990.

${ }^{13}$ K. Andersson, P.-Å. Malmqvist, and B. O. Roos. J. Chem. Phys., 96:1218, 1992

${ }^{14}$ D. Ma, G. Li Manni, J. Olsen, and L. Gagliardi. Journal of Chemical Theory and Computation, 12:3208-3213, 2016.

${ }^{15}$ R. B. Murphy and R. P. Messmer. Chem. Phys. Letters, 183:443, 1991.

${ }^{16}$ K. Wolinski and P. Pulay. J. Chem. Phys., 90:3647, 1989.

${ }^{17}$ E. Rosta and P. R. Surján. J. Chem. Phys., 116:878, 2002.

${ }^{18}$ E. Xu and S. Li. J. Chem. Phys., 139, 2013.

${ }^{19}$ U. Mahapartra, B. Datta, and D. Mukherjee. Chem. Phys. Letters, 299:42-50, 1999.

${ }^{20}$ U. Mahapartra, B. Datta, and D. Mukherjee. Chem. Phys. Letters, 301:206, 1999.

${ }^{21} \mathrm{P}$. Ghosh, S. Chattopadhyay, D. Jana, and D. Mukherjee. Int. J. Mol. Sci., 3:733-754, 2002.

${ }^{22}$ Z. Rolik, Á. Szabados, and P. R. Surján. J. Chem. Phys., 119:1922, 2003.

${ }^{23}$ Á. Szabados, Z. Rolik, G. Tóth, and P. R. Surján. J. Chem. Phys., 122:114104, 2005.

${ }^{24}$ P. Surján, Z. Rolik, Á. Szabados, and D. Kőhalmi. Ann. Phys. (Leipzig), 13:223-231, 2004.

${ }^{25}$ M. Kobayashi and Á. Szabados and H. Nakai and P. R. Surján. J. Chem. Theory. Comput., 6:2024, 2010.

${ }^{26}$ T. Arai. J. Chem. Phys., 33:95, 1960.

${ }^{27}$ P. R. Surján. Topics in current chemistry, 203:63-88, 1999.

${ }^{28}$ V. A. Rassolov. J. Chem. Phys., 117:5978, 2002.

${ }^{29}$ V. A. Rassolov and F. Xu. J. Chem. Phys., 126:234112, 2007.

${ }^{30}$ V. A. Rassolov, F. Xu, and S. Garashchuk. J. Chem. Phys., 120:10385, 2004.

${ }^{31}$ K. V. Lawler, D. W. Small, and M. Head-Gordon. J. Phys. Chem. A, 114:2930-2938, 2010.

${ }^{32}$ G. J. O. Beran, B. Austin, A. Sodt, and M. Head-Gordon. J. Phys. Chem. A, 109:9183-9192, 2005.

${ }^{33}$ P. Surján, P. Jeszenszki, and A. Szabados. Molecular Physics, 113:2960-2963, 2015.

${ }^{34}$ P. Jeszenszki, P. Surján, and Á. Szabados. J. Chem. Theor. Comput., 11:3096-3103, 2015

${ }^{35}$ Z. E. Mihálka, P. R. Surján, and Á. Szabados. J. Chem. Theory Comput., 16:892-903, 2020.

${ }^{36}$ V. A. Rassolov and F. Xu. J. Chem. Phys., 127:044104, 2007.

${ }^{37}$ I. Mayer. Advances in Quantum Chemistry, 12:189-262, 1980.

${ }^{38}$ G. E. Scuseria, C. A. Jiménez-Hoyos, T. M. Henderson, K. Samanta, and J. K. Ellis. J. Chem. Phys., 135(12):124108, 2011. 
${ }^{39}$ Y. Qiu, T. M. Henderson, and G. E. Scuseria. The Journal of Chemical Physics, 145:111102, 2016.

${ }^{40}$ Y. G. Smeyers. An. Fis., 67:17, 1971.

${ }^{41}$ H.-Z. Ye and T. Van Voorhis. Journal of Chemical Theory and Computation, 15(5):2954-2965, 2019.

${ }^{42}$ J. M. Bofill and P. Pulay. The Journal of Chemical Physics, 90:36373646, 1989.

${ }^{43}$ Y. G. Smeyers and L. Doreste-Suarez. International Journal of Quantum Chemistry, 7:687-698, 1973.

${ }^{44}$ J. O. Hirschfelder and R. Silbey. J. Chem. Phys., 45:2188, 1966.

${ }^{45}$ J. N. Murrell and G. Shaw. J. Chem. Phys., 46:1768, 1967.

${ }^{46}$ A. Van der Avoird. J. Chem. Phys., 47:3649, 1967.

${ }^{47}$ B. Jeziorski, K. Szalewicz, and G. Chałasiński. Int. J. Quantum. Chem., 14:271-287, 1978.
${ }^{48}$ I. I. Ukrainsky. International Journal of Quantum Chemistry, 6(3):473-489, 1972.

${ }^{49}$ I. Mayer and M. Kertész. International Journal of Quantum Chemistry, 10(6):961-966, 1976.

${ }^{50}$ P. Epstein. Phys. Rev., 28:695, 1926.

${ }^{51}$ R. Nesbet. Proc. Roy. Soc. (London) Ser. A, 230:312, 1955.

${ }^{52}$ D. E. Woon and T. H. Dunning Jr. J. Chem. Phys., 103:4572, 1995.

${ }^{53}$ D. Földvári, Z. Tóth, P. R. Surján, and Á. Szabados. The Journal of Chemical Physics, 150:034103, 2019.

${ }^{54}$ T.-C. Jagau and J. Gauss. J. Chem. Phys., 137:044116, 2012.

${ }^{55}$ L. V. Slipchenko and A. I. Krylov. The Journal of Chemical Physics, 117:4694-4708, 2002

${ }^{56}$ M. Ecker-Maksić, M. Vazdar, M. Barbatti, H. Lischka, and Z. B. Maksić. J. Chem. Phys., 125:064310, 2006. 


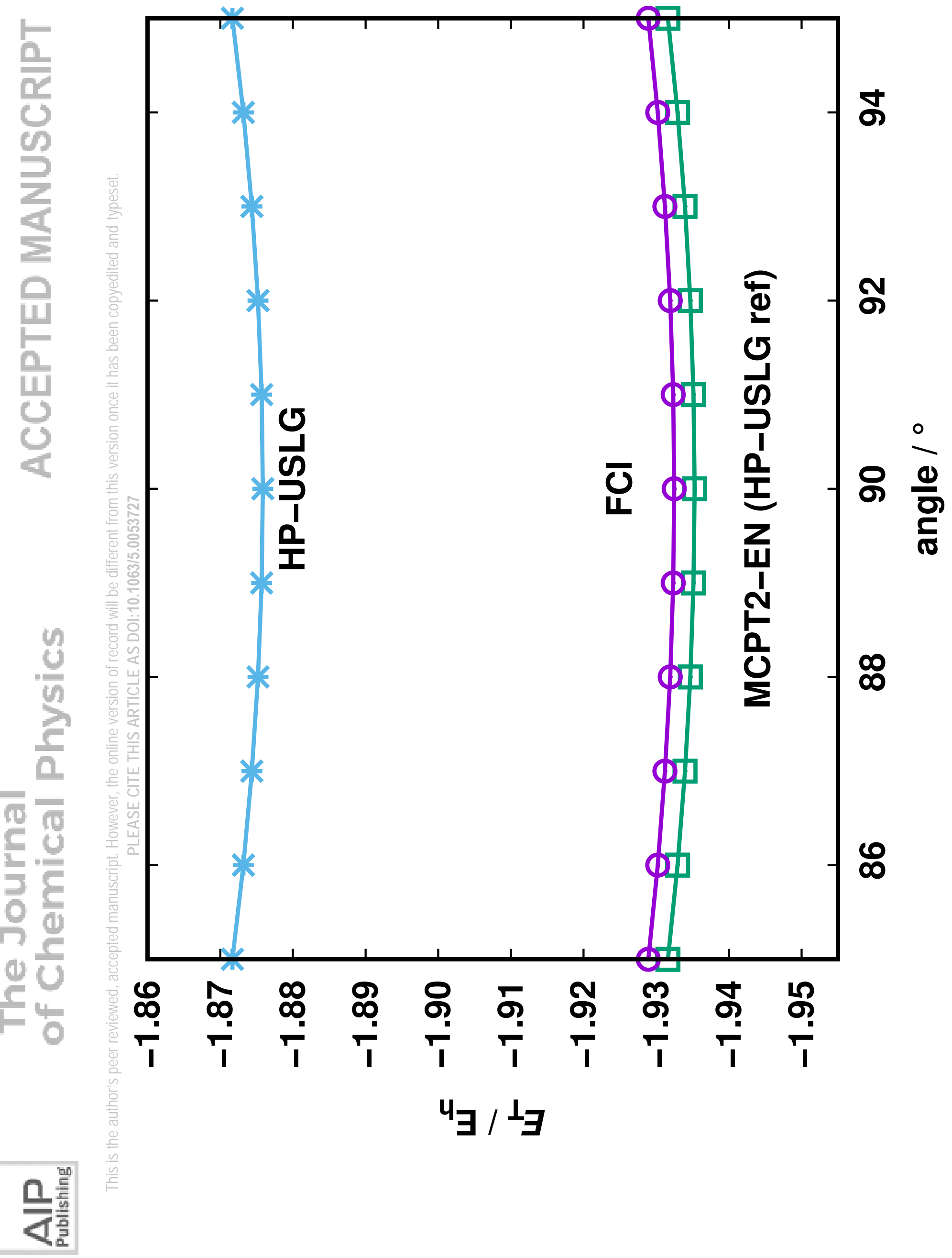




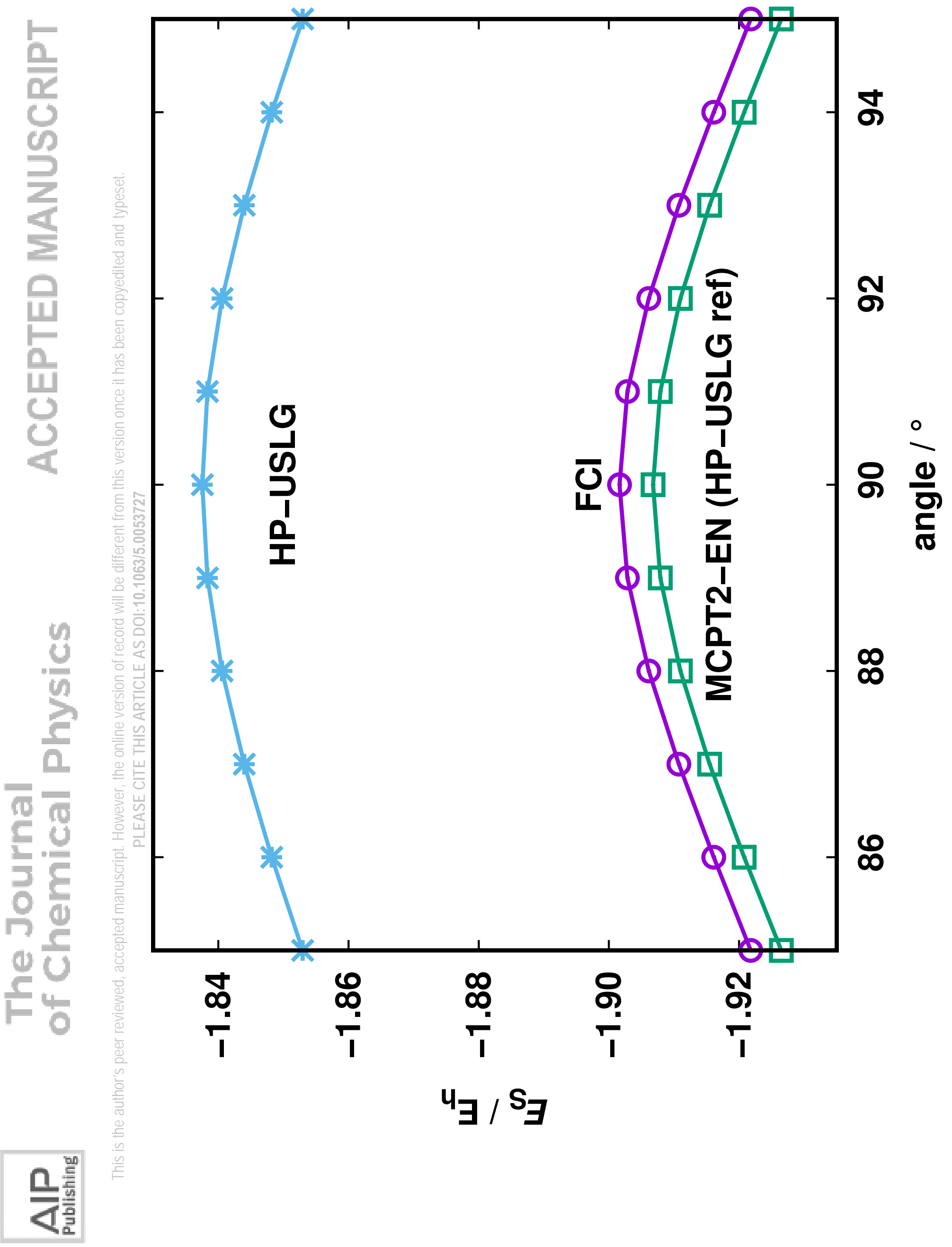




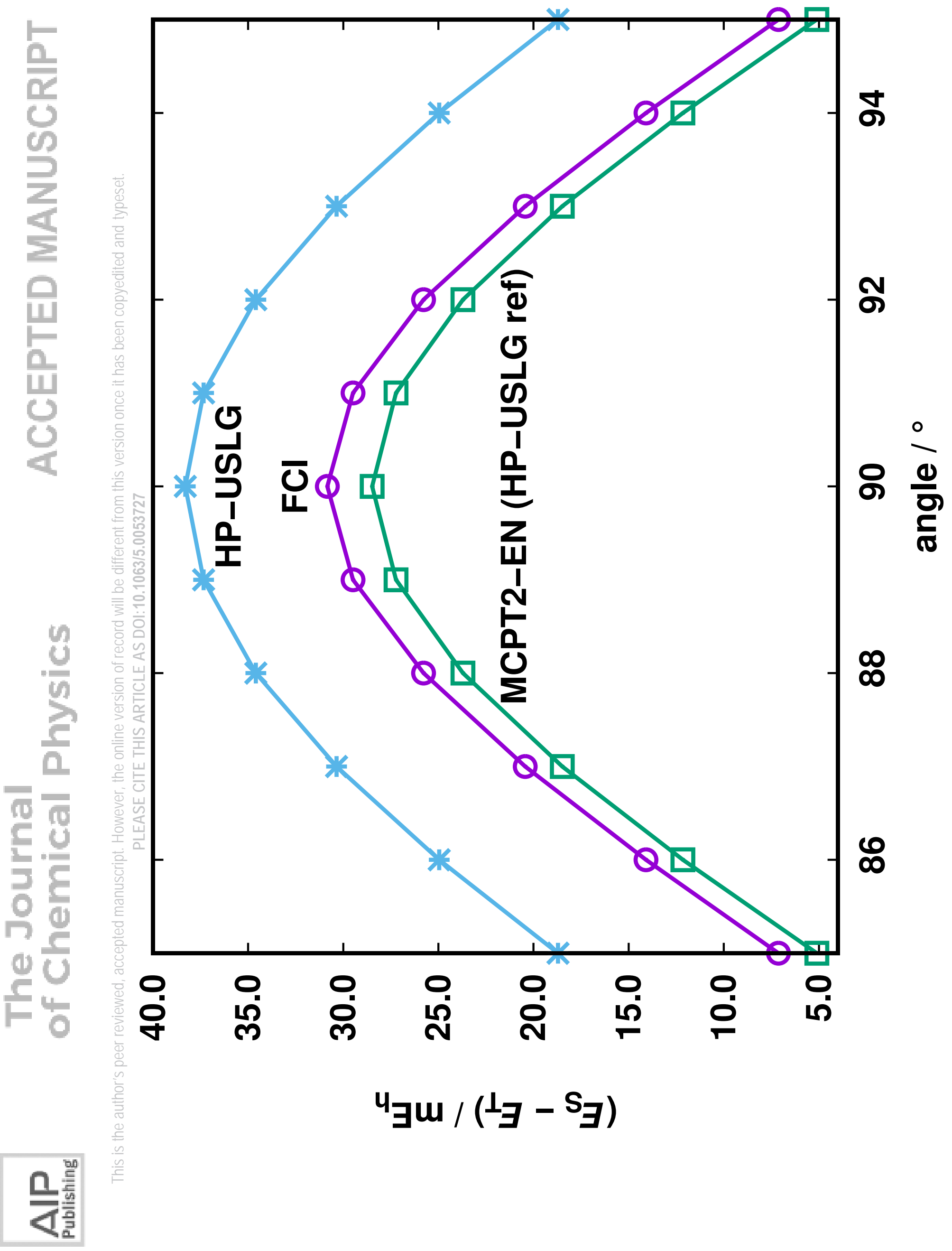

